\title{
Radiologically isolated syndrome is antiquated amidst evolving McDonald criteria for multiple sclerosis - ERRATUM
}

\section{Erratum}

Cite this article: Avasarala $\mathrm{J}$, and Yousuf $\mathrm{F}$ (2021). Radiologically isolated syndrome is antiquated amidst evolving McDonald criteria for multiple sclerosis - ERRATUM. CNS Spectrums 26(4), 436

https://doi.org/10.1017/\$1092852920001790

\section{Jagannadha Avasarala and Fawad Yousuf}

DOI: https://doi.org/10.1017/S1092852919001202; published online by Cambridge University Press 15 July 2019

In the initial publication of Avasarala and Yousuf (2019), the article type was listed incorrectly. The correct article type is Editorial.

The original publication has been corrected to reflect this change.

\section{References}

Avasarala, J., \& Yousuf, F. Radiologically isolated syndrome is antiquated amidst evolving McDonald criteria for multiple sclerosis. Published online 15 July 2019. CNS Spectrums, doi:10.1017/S1092852919001202. 